УДК 574; 502.37

ИССЛЕДОВАНИЕ ИНФОРМАЦИОННЫХ ХАРАКТЕРИСТИК

ФЛУОРЕСЦЕННОГО МЕТОДА ДИАГНОСТИКИ СТЕПЕНИ

ЗАГРЯЗНЕННОСТИ ПОЧВЫ НЕФТЕПРОДУКТАМИ

\title{
INVESTIGATION OF FLUORESCIAL METHOD INFORMATION CHARACTERISTICS FOR DIAGNOSTICS OF THE DEGREE OF SOIL POLLUTION BY OIL PRODUCTS
}

\author{
А.Ф. Ахмедов \\ Научно-исследовательский институт аэрокосмической информатики \\ Национального аэрокосмического агентства, \\ г. Баку, Азербайджанская Республика \\ Arshad Farhad oglu Ahmadov \\ Research Institute of Aerospace Informatics, \\ National Aerospace Agency, \\ Baku, Azerbaijan Republic \\ e-mail: asadzade@rambler.ru
}

\begin{abstract}
Аннотация. Статья посвящена исследованию информационных характеристик флуоресцентного метода диагностики степени загрязненности почвы нефтепродуктами. Показано, что метод флуоресцентного зондирования, широко используемый для определения степени загрязнения почвы нефтью и нефтепродуктами, может быть реализован в оптимальном режиме в смысле достижения высокой информативности результатов измерения в зоне, загрязненной нефтепродуктами и нефтью, состоящей из эквивалентных подзон, загрязненных пространственно негомогенно при выполнении следующих условий: (а) исследуется множество подзон, степень загрязненности
\end{abstract}


которых априорно одинакова, (б) исследование подзон должно быть осуществлено при разных длинах волн; (в) количество отсчетов должно быть неодинаковым по разным подзонам; (г) реализуется особый порядок выбора индикационного показателя, длины волны возбуждающего излучения и количество отсчетов, при котором в одной из подзон может быть достигнута максимальная информативность.

Abstract. The article is devoted to the research of the information characteristics of the fluorescent method for diagnosing the degree of soil pollution by oil products. It is shown that the method of fluorescent sensing, widely used to determine the degree of soil pollution by oil and oil products, can be implemented optimally in the sense of achieving high informativeness of measurement results in the zone polluted by oil products and oil, consisting of equivalent subzones polluted spatially inhomogeneous under conditions: (a) many subzones are investigated, the pollution degree of which is a priori the same, (b) the subzones should be investigated at different wavelengths; (c) the number of counts should be different in different subzones; (d) a special procedure is implemented for selecting the indicator, the wavelength of the exciting radiation, and the number of samples in which the maximum information content can be achieved in one of the subzones.

Ключевые слова: почва, нефтепродукты, загрязнение, флуоресцентный метод, оптимизация, информация

Key words: soil, oil products, pollution, fluorescent method, optimization, information

Хорошо известно, что флуоресцентный метод зондирования загрязненных нефтяными углеводородами сред обладает рядом таких преимуществ, как возможность получения высокоточной информации в реальном времени и высокая информативность. Будучи малозатратным и 
высокочувствительным методом, флуоресцентное зонирование широко используется при исследовании водных пространств и земельных участков на предмет загрязнения нефтяными углеводородами.

Метод флуоресцентного зондирования основан на хорошо исследованном факте наличия сильной корреляционной зависимости между химическим составом и плотностью нефти с такими физическими показателями флуоресцентного излучения, как интенсивность излучения, распределение интенсивности по длине волны, время свечения. В работе [1] исследована возможность использования флуоресцентного метода для определения градуса API, являющегося широко применяемым показателем плотности сырьевой нефти. В работе [2] показано, что метод флуоресцентного зондирования также позволяет исследовать содержание фитопланктонов и растворенного органического вещества в водной поверхности. Согласно [2, 3], для исследования загрязненности водной поверхности различными органическими загрязнителями могут быть использованы лазерные или узконаправленные оптические лучи на длинах волн 308, 355, 532 и 266 нм. При таком широком спектре углеводородных загрязнителей, которые могут быть исследованы флуоресцентными методами, предъявляются особые требования для проведения исследований информативности этого метода. Дело в том, что для исчерпывающего анализа информативности этого метода должны быть приняты во внимание следующие физические показатели процесса флуоресцентного зондирования:

1. Длина волны возбуждающего оптического излучения;

2. Длина волны сгенерированного флуоресцентного излучения;

3. Совместное рассмотрение флуоресцентной излучательной способности как сырьевой нефтяной продукции, так и ее отдельных составляющих или переработанной нефтяной продукции.

Как отмечается в работе [4], в 2006 году в Австралии были проведены экспериментальные исследования, наиболее полно соответствующие 
вышеуказанным требованиям. Кратко изложим суть проводимых в [4] экспериментальных исследований. Исследованию подвергались следующие углеводороды:

- типы нефти, добываемые в Австралии:

1. Corallina -1 ,

2. Dockrell - 2,

3. Juabert - I,

4. Enfield - I;

- нефтепродукты:

5. бензин без свинцовой добавки,

6. дизель,

7. моторное масло.

Использовались следующие длины волн: 420, 490 и 575 нм. Для осуществления классификации углеводородов были вычислены следующие показатели:

$$
\begin{aligned}
& I_{B-Y}=\frac{I_{420}}{I_{575}}, \\
& I_{G-Y}=\frac{I_{490}}{I_{575}},
\end{aligned}
$$

где $I_{\lambda}-$ измеренная величина интенсивности флуоресцентного излучения на длине волны $\lambda$.

Графики показателей $I_{B-Y} \quad$ и $\quad I_{G-Y}$ применительно к случаям использования возбуждающего излучения на длинах волн 375, 385, 395 и 405 нм приведены на рисунках 1 и 2. 


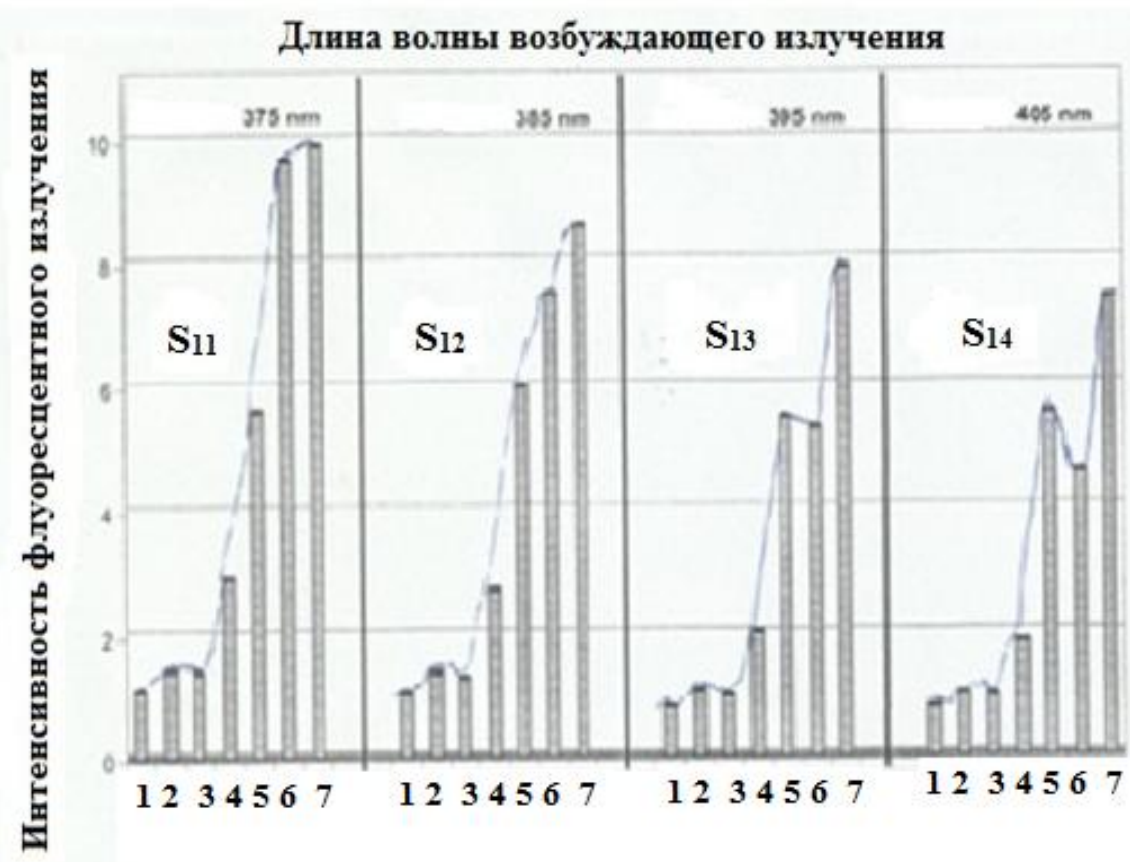

Тип нефти и нефтепродуктов

Принятые обозначения: $\mathrm{S}_{1 \mathrm{i}} i=\overline{1,4}$ - площадь фигуры, находящейся под соответствующей пунктирной линии

Рисунок 1 . Диаграммы показателей $I_{B-Y}$ для различных углеводородов при разных длинах волн возбуждающего излучения

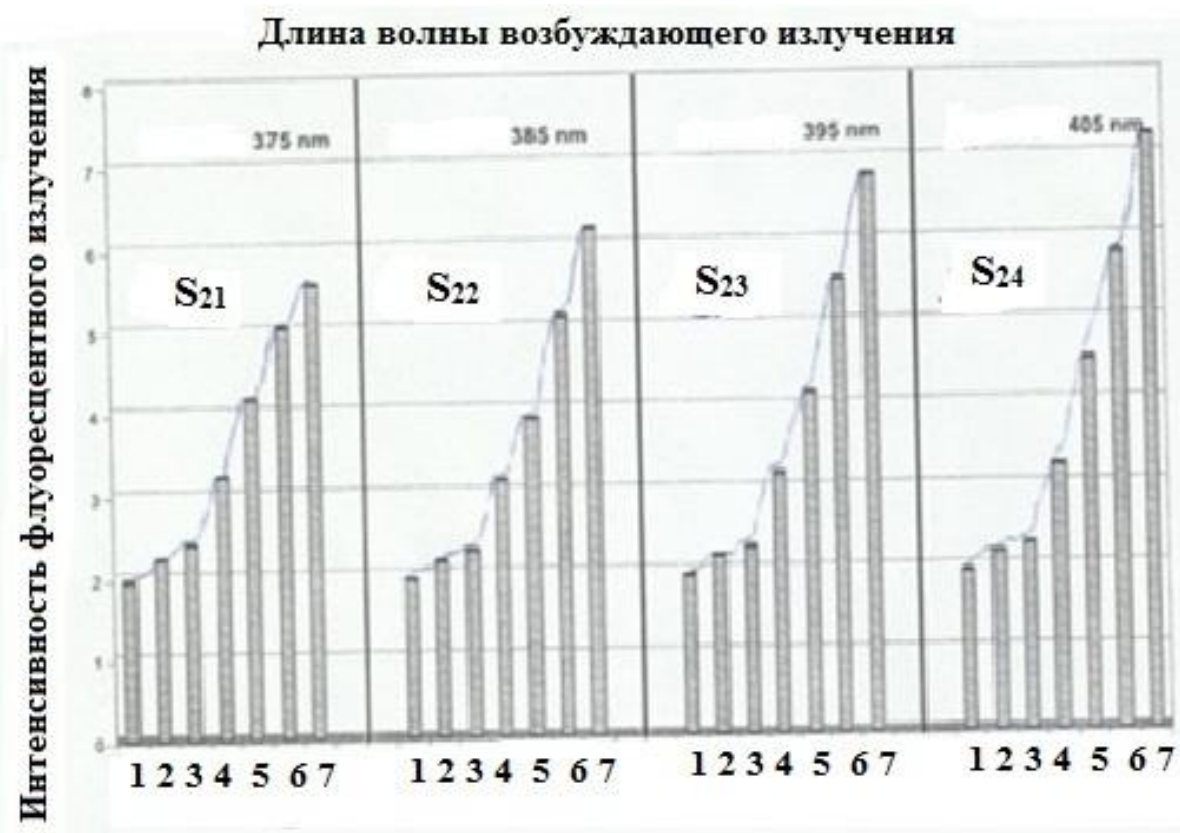

Тип нефти и нефтепродуктов

Принятые обозначения: $\mathrm{S}_{2 \mathrm{i}} i=\overline{1,4}-$ площадь фигур, находящихся под соответствующей пунктирной линией

Рисунок 2 .Диаграммы показателей $I_{G-Y}$ для различных углеводородов при разных длинах волн возбуждающего излучения 
Результаты экспериментальных исследований, проведенных в [4], позволяют сделать следующие выводы:

1. Флуоресцентные свойства определенного множества углеводородов, представленных на рисунках 1 и 2, по оси абсцисс могут быть охарактеризованы суммарным показателем площади, находящейся под соответствующей пунктирной линии $\mathrm{S}_{\mathrm{ji}}$, где $\mathrm{j}=\overline{1,2} ; i=\overline{1,4}$. Здесь индекс $j$ определяет использование показателей (1) или (2), а индекс $i$ определяет длину волны возбуждающего излучения.

2. С ростом $i$ при $j=1$ наблюдаются уменьшение показателя $\mathrm{S}_{1 \mathrm{i}}$ и увеличение показателя $\mathrm{S}_{2 \mathrm{i}}$.

3. Значения $\mathrm{S}_{\mathrm{ji}}$ при фиксированном $i$ и при разных $j$ противофазны, т.е. если $S_{1 \mathrm{i}}$ больше, то $S_{2 \mathrm{i}}$ меньше, и наоборот.

На основе вышеизложенных особенностей результатов флуоресцентного зондирования различных типов углеводородов рассмотрим предлагаемую модель исследования загрязненности почв нефтепродуктами:

1. Зона, загрязненная нефтепродуктами и нефтью, состоит из эквивалентных подзон, загрязненных пространственно негомогенно (рисунок 3);

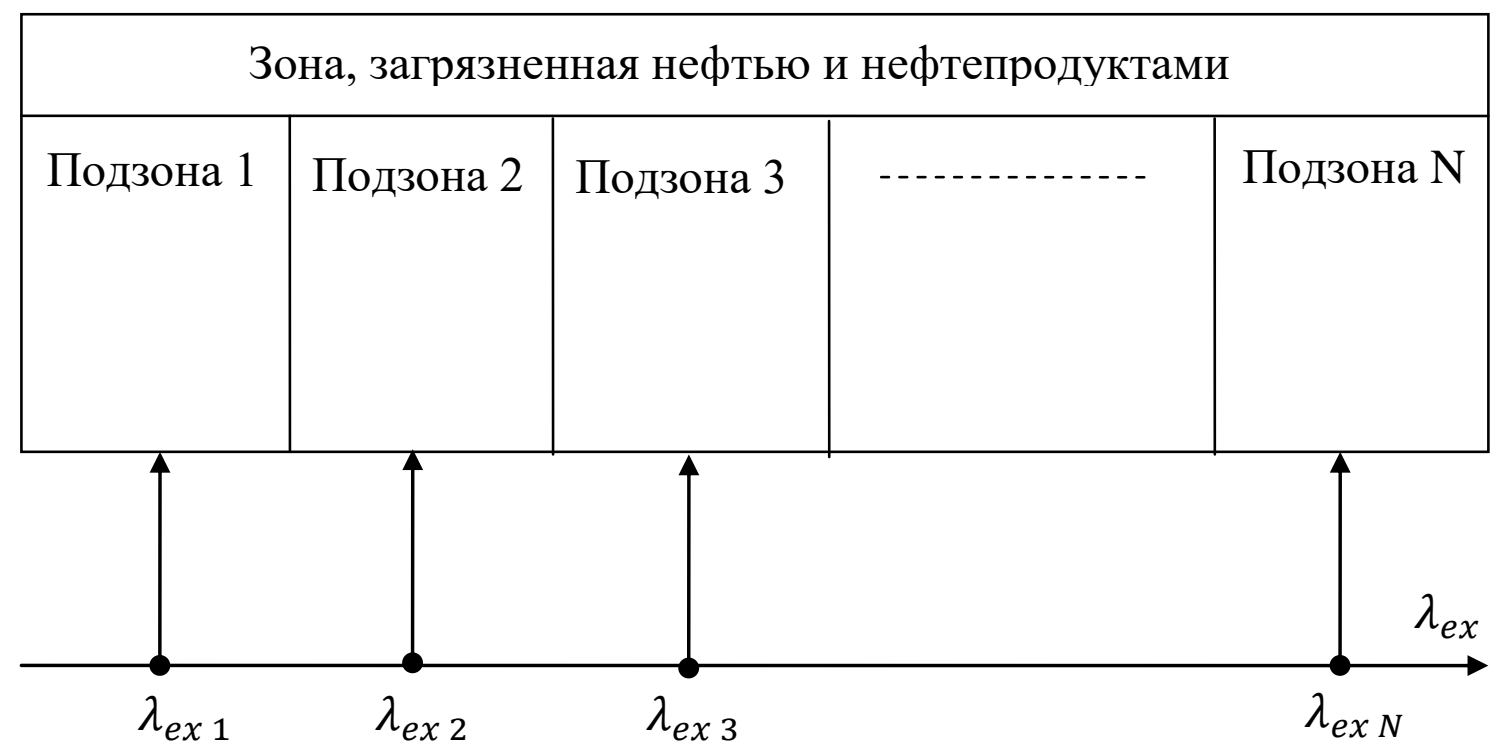

Рисунок 3. Модель исследования степени загрязненности подзон методом флуоресцентного зондирования 
2. Так как подзоны эквиваленты по степени загрязнения, то для исследования условий достижения наибольшей информативности в $i$-ой подзоне применяется возбуждающее излучение только с длиной волны $\lambda_{e x . I}$, где $i=\overline{1, n}$;

3. Так как подзоны негомогенны, при проведении $\mathrm{N}$ числа измерений в подзоне можно получить $\mathrm{N}$ количество не равных отчетов. При этом максимальная величина отчета с $i$-ой подзоны определяется как $\mathrm{V}_{\max . \mathrm{i}}$ $V_{\text {max.i }}\left(\lambda_{\text {exi }}\right)$. Возможное количество градаций загрязненности $i$-ой подзоны определяется

$$
M_{i}=\frac{U_{\operatorname{maxi}}\left(\lambda_{\text {exi }}\right)}{\Delta \mathrm{U}}
$$

$\Delta \mathrm{U}$ - квант сигнала.

4. Информация, получаемая в результате проведения $\mathrm{N}_{\mathrm{i}}$ количества измерений в $i$-ой подзоне, определяется как

$$
M_{i}=N_{i} \cdot \log _{2} \frac{U_{\operatorname{maxi}}\left(\lambda_{e x i}\right)}{\Delta \mathrm{U}} .
$$

5. Допускается, что $\mathrm{N}_{\mathrm{i}}$ определяется в зависимости от $\lambda_{\text {i, }}$ т.е. существует функциональная зависимость

$$
N_{i}=\varphi\left(\lambda_{\text {exi }}\right)
$$

6. Первый функционал цели определяется как

$$
\begin{array}{r}
F_{g}=\sum_{i=1}^{n} \varphi\left(\lambda_{\text {exi }}\right) \cdot \log _{2} \frac{U_{\operatorname{maxi}}\left(\lambda_{\text {exi }}\right)}{\Delta \mathrm{U}}(\text { в дискретном виде), } \\
F_{g}=\int_{0}^{\lambda_{\max }} \varphi\left(\lambda_{e x}\right) \cdot \log _{2} \frac{U_{\operatorname{maxi}}\left(\lambda_{e x}\right)}{\Delta \mathrm{U}} d \lambda \text { (в непрерывном виде). }
\end{array}
$$

Применительно к функциональному (7) допускаются следующие типы зависимости $U_{\max }=U_{\max }\left(\lambda_{e x}\right)$ :

$$
\begin{aligned}
& U_{\max }=U_{\max 01}+k_{1} \lambda_{e x}, \\
& U_{\max }=U_{\max 02}+k_{2} \lambda_{e x} .
\end{aligned}
$$


Целью оптимизации по функционалу (7) является отыскание оптимальной величины $\left(\lambda_{e x}\right)$.

Далее допускаются следующие типы зависимости:

$$
\begin{gathered}
N_{i}=\varphi(\lambda i), \\
\varphi\left(\lambda_{\text {exi }}\right)=\varphi_{01}+d_{1} \cdot \lambda_{\text {exi }} \\
\varphi\left(\lambda_{\text {exi }}\right)=\varphi_{02}+d_{2} \cdot \lambda_{\text {exi }} .
\end{gathered}
$$

С учетом функционала (7) эвристически понятно, что экстремум интегранта в (7) может появиться при выборе следующих пар выражений: [(8), (11)] или [(9), (10)]. Нетрудно показать, что при выборе пары выражений [(8), (11)] оптимальная величина длины волны возбуждающего излучения может быть определена путем решения следующего трансцендентного уравнения:

$$
\log _{2}\left[\frac{\left(U_{\max 01}+k_{1} \lambda_{e x}\right)}{\Delta U}\right]=\frac{\left(U_{02}-d_{2} \cdot \lambda_{e x}\right)}{d_{2}} \cdot \frac{k_{1}}{\left(U_{\max 01}+k_{1} \lambda_{e x}\right) \cdot \ln 2} .
$$

При выборе пары выражений [(9), (10)] оптимальная величина длины волны возбуждающего излучения может быть определена путем решения следующего трансцендентного уравнения:

$$
\log _{2}\left[\frac{\left(U_{\max 02}-k_{2} \lambda_{e x}\right)}{\triangle U}\right]=\frac{\left(U_{01}+d_{1} \cdot \lambda_{e x}\right)}{d_{2}} \cdot \frac{k_{2}}{\left(U_{\max 02}-k_{2} \lambda_{e x}\right) \cdot \ln 2} .
$$

Можно показать, что условия (12), (13) определяют те экстремальные значения $\lambda_{e x}$, при которых количество информации, полученной при проведении измерений в соответствующей подзоне, достигает максимального значения.

Таким образом, с учетом вышеизложенного можно предложить алгоритм выбора подзон, результаты измерений в которых оказываются наиболее информативными.

Блок-схема алгоритма представлена на рисунке 4. 


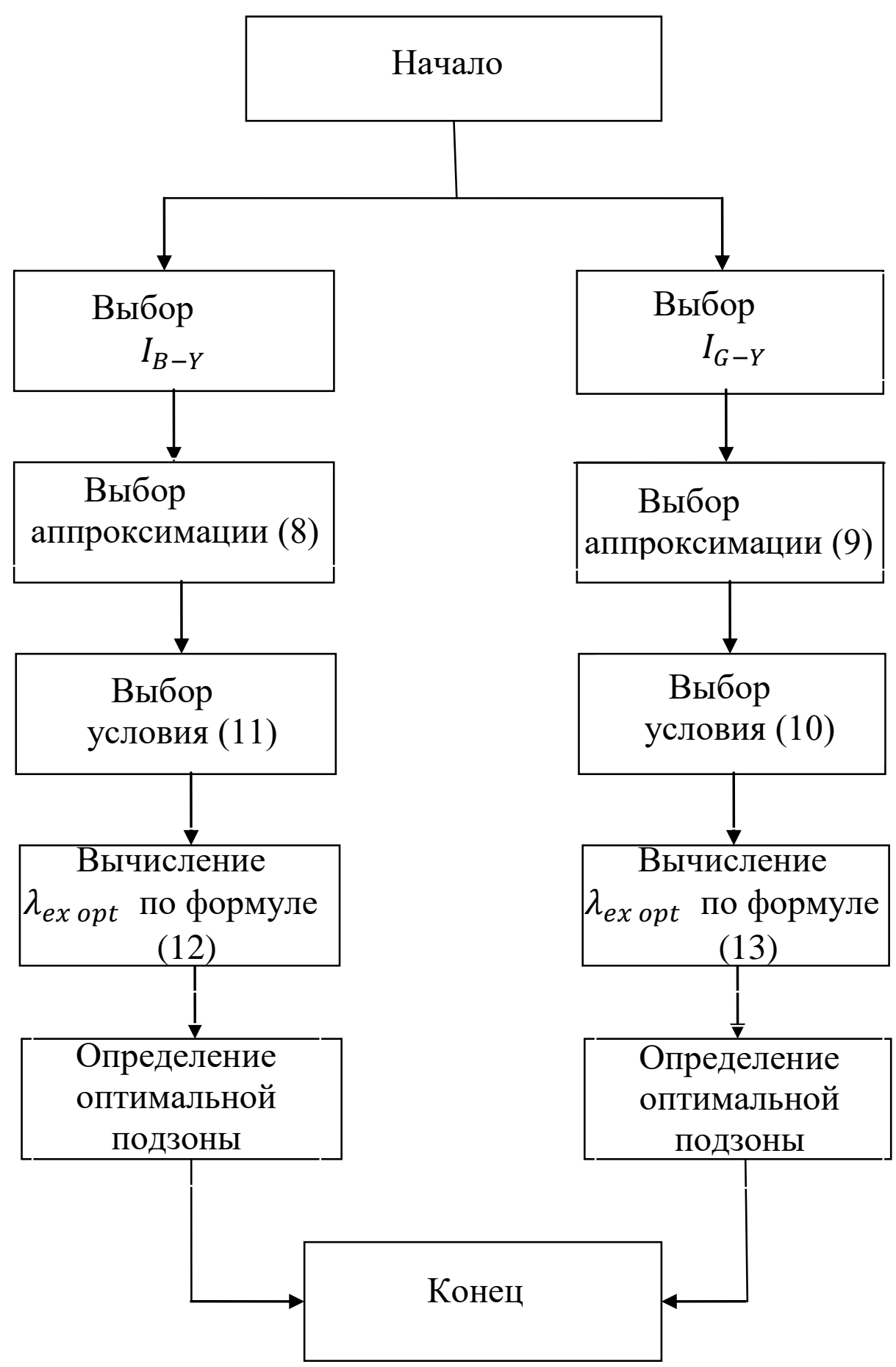

Рисунок 4. Блок-схема алгоритма выбора наиболее информативной подзоны при исследовании загрязненных нефтепродуктами почв методом флуоресцентного зондирования 


\section{Вывод}

Таким образом, показано, что метод флуоресцентного зондирования, широко используемый для определения степени загрязнения почвы нефтью и нефтепродуктами, может быть реализован в оптимальном режиме в смысле достижения высокой информативности результатов измерения в зоне, загрязненной нефтепродуктами и нефтью, состоящей из эквивалентных подзон, загрязненных пространственно негомогенно при выполнении следующих условий: (а) исследуется множество подзон, степень загрязненности которых априорно одинакова, (б) исследование подзон должно быть осуществлено при разных длинах волн; (в) количество отсчетов должно быть неодинаковым по разным подзонам; (г) реализуется особый порядок выбора индикационного показателя, длины волны возбуждающего излучения и количества отсчетов, при котором в одной из подзон может быть достигнута максимальная информативность.

\section{Список используемых источников}

1. Fder A.G., İwanski P., Montanari L. Light Emissions from Oil: A Vaulable İnoformation source for ppetrolium analysis, Tpoint, \#2? P. 9-14, 2004.

2. Chubarov V.V., Fadeev V.V. Ecological Monitoring in the Caspian Sea (Mouth Zone of the River Volga) with a Shipboard Laser Spectrometr, EARSeL eProceedings 3, 2004.

3. Patsayva S., Yuzhakov V., Varlamov V., Barbini R., Fantoni R., Frassanito C. And Palucci A. Laser Spectroskopy of Mineral Oils on the Water Surface // Proceedings of EARSeL - SIG -Workshop, 2000.

4. Rostampour V., Lynch M.J. Quantitative Techniques to Discriminate Petroleum Oils Using LED - Induced Fluorescence. www.witpress.com, ISSN 1743-3541 (on-line). 2006 WIT Press Water Pollution VIII: Modeling, Monitoring and Management 255 WIT Transactions on Ecology and the Environment, Vol. 95, DOI:10.2495/WP060261. 


\section{References}

1. Fder A.G., İwanski P. and Montanari L. Light Emissions from Oil: A Vaulable İnoformation source for ppetrolium analysis. Tpoint, 2004, pp. 9-14.

2. Chubarov V.V., Fadeev V.V. Ecological Monitoring in the Caspian Sea (Mouth Zone of the River Volga) with a Shipboard Laser Spectrometr. EARSeL eProceedings, 2004, Vol. 3.

3. Patsayva S., Yuzhakov1 V., Varlamov V., Barbini R., Fantoni R., Frassanito C., Palucci A. Laser Spectroskopy of Mineral Oils on the Water Surface. Proceedings of EARSeL-SIG-Workshop, 2000.

4. Rostampour V., Lynch M.J. Quantitative Techniques to Discriminate Petroleum Oils using LED - Induced Fluorescence. WIT Press Water Pollution VIII: Modeling, Monitoring and Management 255 WIT Transactions on Ecology and the Environment. 2006, Vol. 95. DOI: 10.2495/WP06026. Available at: www.witpress.com.

\section{Сведения об авторе}

\section{About the author}

Ахмедов Аршад Фархад оглы, начальник научно-технического отдела Научно-исследовательского института аэрокосмической информатики Национального аэрокосмического агентства, г. Баку, Азербайджанская Республика

Ahmadov Arshad Farhad oglu, Head of Scientific-Technical Department of Research Institute of Aerospace Informatics of National Aerospace Agency, Baku, Azerbaijan Republic

e-mail: asadzade@rambler.ru 